\title{
Uber Schmerzen bei Encephalitis epidemica. Ein Beitrag zur Kenntnis der zentralen Schmerzen ${ }^{1}$ ). \\ Von \\ Willibald Sauer (München).
}

Mit 3 Textabbildungen.

(Eingegangen am 18. Mai 1922).

Wenn man die zahllosen Veröffentlichungen über die Encephalitis epidemica überblickt, findet man in ihnen zwar die eingehendsten Beschreibungen der bei ihr beobachteten Motilitätsstörungen, man wird aber vergeblich nach näheren Angaben über Schmerzen, wie über Sensibilitätsstörungen überhaupt suchen. In der Regel werden sie gar nicht erwähnt. Schon dieser Umstand allein deutet darauf hin, daß Schmerzen bei der Encephalitis epidemica eine Seltenheit sind. Und doch kommen sie gelegentlich in allen Abschnitten der Erkrankung vor. Am häufigsten wohl, auch wenn man von dem bekannten initialen Kopfschmerz absieht, im akuten Stadium. Nur ist ihre Deutung gerade während desselben wegen der oft vorhandenen Bewußtseinstrübung und der motorischen Unruhe bei den hyperkinetischen Formen mit großen Schwierigkeiten verknüpft, wenn nicht ganz unmöglich. Als Untersuchungsmaterial kommen daher in erster Linie die chronischen Fälle in Betracht.

Bezüglich der Auffassung der Schmerzen stehen sich zwei diametral entgegengesetzte Ansichten gegenüber. Die einen halter sie für periphere, die anderen, wie besonders $S t e r t z^{2}$ ), für zentrale. Man geht wohl nicht fehl, wenn man heute behauptet, daß die Häufigkeit peripherer Schmerzen bei der Encephalitis jedenfalls bei weitem überschätzt worden ist.

Daß die Entscheidung der Frage peripher oder zentral so große Schwierigkeiten macht, dürfte vor allem darauf zurückzuführen sein, daß unsere Kenntnis der zentralen Schmerzen überhaupt auch heute noch eine sehr dürftige ist. Es fehlt zwar nicht an einwandfreien

1) Nach einem am 7. II. 1922 in der Münchener Neurologisch-Psychiatrischen Gesellschaft gehaltenen Vortrage.

2) Ärztl. Verein München, Sitzung vom 24. IrI. 1920. Münch. med. Wochenschr. 1920, S. 467. - Sitzung d. Deutsch. Forsch. - Anstalt f. Psych, v. 20. V. 1920. 1920. Zeitschr. f. d. ges. Neurol. u. Psychiatr. Ret. 21, 363/4. 
Beobachtungen, die ihre Existenz sicherstellen, auch nicht an Beschreibungen, die sie in ihrer Eigenart näher zu kennzeichnen suchen. Im großen und ganzen sind es aber doch mehr negative oder indirekte Momente, die zu ihrer Erkennung herhalten müssen, also in erster Linie der Ausschluß einer peripheren Erkrankung, in zweiter der Nachweis einer zentralen Affektion. Falls letzterer nicht oder noch nicht zu führen ist, war man bisher fast ausschließlich auf das erstere Moment angewiesen. Es scheint nun, daß die Encephalitis epidemica, die für uns Neurologen und Psychiater in so mannigfacher Beziehung zu einer über Erwarten ergiebigen Fundgrube geworden ist, auch in dieser Hinsicht uns ein Stück weiter zu bringen berufen ist. Die Beobachtungen, über die ich im folgenden berichten möchte, weisen darauf hin, daß es bei der Encephalitis epidemica einen Schmerztypus gibt, der, wenn vorhanden, die Frage peripher oder zentral mit Sicherheit zu entscheiden erlaubt und, wie es scheint, für die Encephalitis epidemica und Erkrankungen ähnlicher Lokalisation sogar pathognomonisch ist.

Im Juni 1921 wurde mir eine 19 jährige Kontoristin wegen heftiger Schmerzen zur psychotherapeutischen Behandlung überwiesen. Das Begleitschreiben, das sie mitbrachte, lautete: „Fräulein F. vor einem Jahre an Kopfgrippe erkrankt, leidet an Schmerzen im linken Unterschenkel. Röntgendurchleuchtung ergab normale Verhältnisse. Zweifellos Hy." Die Patientin selbst gab an, daß sie an ununterbrochenen, Tag und Nacht anhaltenden Schmerzen im linken Unterschenkel leide, die oberhalb des inneren Knöchels beginnen und bis zur Fußsohle hinabziehen. Die Schmerzen werden von ihr als stoßweises Reißen und Stechen gekennzeichnet. Ihr auffälligstes Merkmal ist aber, daß sie nur im Sitzen, Liegen und im Stehen vorhanden sind. Beim Gehen spüre sie nichts. Auch jede sonstige Bewegung des Fußes bringe die Schmerzen sofort zum Verschwinden. Ferner habe sie keine Schmerzen, wenn sie aufhöre zu atmen. Bei einer späteren Exploration gab sie noch an, daß die Schmerzen auch dann leichter werden, wenn sie ganz tief einatme und darauf ganz kurz ausatme, was wohl mit einem vorübergehenden Atemstillstand gleichbedeutend ist. Desgleichen beim Sprechen, weil sie dann auch weniger atme. Manchmal habe sie auch Zittern im linken Bein, jedoch ebenfalls nur in der Ruhe, beim Gehen spüre sie nichts davon. Temperaturwechsel habe auf die Schmerzen keinen Einfluß. Infolge ihrer Heftigkeit sei die Nachtruhe in hohem Maße gestört.

Diese Schmerzen bestehen seit der „Kopfgrippe“, an der sie im März 1920 erkrankte. Sie war angeblich 10 Tage bewußtlos, war anfangs an Armen und Beinen vollständig gelähmt und hatte fürchterliche Kopfschmerzen. Als sie nach 7 Wochen aus dem Krankenhause ent- 
lassen wurde, habe sie so schnell gesprochen, daß niemand sie verstehen konnte. Ähnliche Schmerzen wie am linken Fuß hatte sie zu Beginn der Krankheit auch an der linken Hand, und zwar nur am Handrücken und an der Hohlhand, während die Finger verschont blieben. Diese Schmerzen hielten jedoch nur zwei bis drei Tage an. Die Patientin gab noch an, daß sie früher viel stärker als jetzt gewesen sei. Sonst habe sie keine Veränderung an sich wahrgenommen. Auf eindringliches Befragen gab sie jedoch zu, daß sie seit der Grippe nicht mehr so lustig sei, während sie früher arg heiter gewesen sei, jetzt dagegen oft traurig werde und oft, ganz ohne Grund, weine.

Aus der Familienanamnese ist zu erwähnen, daß die Mutter sehr hastig und nervös ist. Sie selbst war früher stets gesund, nur seit jeher leicht erregbar.

Wegen der Schmerzen stand Patientin bei gut einem halben Dutzend Ärzten in Behandlung, wurde elektrisiert, massiert, mit Einreibungen, Sonnenbädern, Moorbädern, Vaccineurin, Arsen, Phosphor und Eisen behandelt. Es war alles vergeblich.

Die Untersuchung der schmächtigen, etwas unterernährten $\mathrm{Pa}$ tientin ergab folgenden Befund: Zunächst rein äußerlich: das eigentümlich gebundene Wesen, Verlangsamung und Erschwerung aller Bewegungen, vor allem ihres Beginnes, die statuenhafte, vornübergeneigte Haltung, den charakteristischen Gang mit kleinen trippelnden Schritten, den zwar nicht starren, aber eigentümlich gespannten Gesichtsausdruck mit dem offensichtlich zwangsmäßigen, unnatürlich lang verharrenden Lachen, ein ausgesprochenes Salbengesicht, die abgerissene, schwer verständliche Sprache, Speichelfluß - alles wohlbekannte Kennzeichen des sog. Pallidumsyndroms. Aus dem sonstigen neurologischen Befunde ist folgendes erwähnenswert: Horizontaler Nystagmus, hauptsächlich beim Blick nach links, aber auch nach rechts (angeblich angeboren), Reflexe an den oberen Extremitäten links viel. leicht etwas lebhafter als rechts; doch ist der Unterschied kein großer. P. S. R. und A. S. R. beiderseits sehr lebhaft, links ebenfalls eine Spur lebhafter als rechts. Babinski links nicht konstant, aber wiederholt deutlich von dem äußeren Rande des linken Fußes auslösbar. Abgesehen von gelegentlichen klonusartigen Zuckungen beider Füße sonst keine spastischen Phänomene. Keine Contracturen, weder rechts noch links. Muskeltonus links vielleicht eine Spur stärker als rechts. Keine Ataxie*). Keine Adiadochokinese. Keine Störung des Lagegefühls.

*) Nachtrag bei der Korrektur: Nur bei der ersten Untersuchung war subjektiv und objektiv eine leichte Unsicherheit beim Fingernasenversuch rechts feststellbar, bei späteren Untersuchungen nicht mehr. Auch der linksseitige Babinski ist seit Monaten nicht mehr auslösbar. 
Bei der Prüfung der Abhängigkeit der Schmerzen von der Bewegung zeigte sich, daß sie nicht nur durch aktive, sondern auch durch passive Bewegungen prompt behoben werden. Und zwar genügen hierzu schon Bewegungen von minimaler Exkursionsbreite. Auch wurde derselbe Effekt erzielt, ob nun die Bewegungen ausschließlich im Fußgelenk oder im Knie- oder Hüftgelenk bei fixiertem Fußgelenk ausgeführt wurden. Die Zeitspanne vom Beginn der Bewegung bis zum Verschwinden der Schmerzen wie umgekehrt bis zu ihrem Wieder. auftreten nach Ruhigstellung des Gliedes betrug jedesmal nur wenige Sekunden.

Entsprechend der Verbreitung der Schmerzen war folgende Sensibilitätsstörung nachweisbar:

Die obere Grenze verläuft horizontal etwa $10 \mathrm{~cm}$ oberhalb des inneren Knöchels, die vordere schneidet mit der vorderen Schienbein-

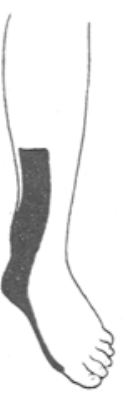

Abb. 1 .

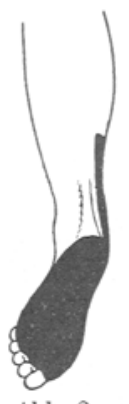

Abb. 2.

Kältehyperästhesie und Hypästhesie für die übrigen Qualitäten. kante $a b$, die hintere verläuft parallel der Achillessehne ein bis zwei Querfinger von ihr entfernt. Über eine etwa handbreite Brücke unterhalb des inneren Knöchels erstreckt sich dann die Sensibilitätsstörung über die ganze Fußsohle mit Ausnahme der Zehen. Innerhalb dieser Grenzen besteht eine Abstumpfung der Sensibilität für sämtliche Qualitäten mit Ausnahme der Kälteempfindlichkeit, die im ganzen Bereich und besonders an der Fußsohle gesteigert ist. Das Unterscheidungsvermögen für spitz und stumpf, das an stärksten betroffen ist, ist am Unterschenkel nahezu aufgehoben.

Diese Vergesellschaftung von Kältehyperästhesie mit Hypästhesie für die übrigen Qualitäten erinnert an Leydens relative Hyperästhesie (Hypästhesie und Hyperalgesie), die nach Lewandowsky ${ }^{1}$ ) bei Kranken mit zentralen Schmerzen regelmäßig gefunden wird. Eine Verlangsamung der Schmerzleitung, die nach Lewandowsky ebenfalls charakteristisch sein soll, war nicht feststellbar. Auch das Lokalisationsvermögen war ungestört.

Die auffällige Abhängigkeit der Schmerzen von der Bewegung und der Atmung kam mir so ungewöhnlich vor, daß ich sie zunächst für eine rein zufällige hielt. Als ich dann gelegentlich meine Aufzeichnungen über früher beobachtete Encephalitiskranke durchmusterte, fand ich einen weiteren Fall, der eine weitgehende Übereinstimmung mit dem erstgenannten darbot.

1) Lewandowsky, Die zentralen Sensibilitätsstörungen. Handbuch 1, 801. 
Es handelte sich um einen 22jährigen Kaufmann, der ebenfalls im März 1920 an „Gehirngrippe" erkrankt war. Auch in diesem Falle setzten die Schmerzen, die in die rechte Wade bzw. an die Innenseite des rechten Unterschenkels verlegt wurden, mit dem Beginn der Erkrankung ein und haben bis zum heutigen Tage angehalten. Auch dieser Kranke, der in noch ausgesprochenerem Maße als die Vorhergenannte alle Zeichen des Parkinsonschen Symptomenkomplexes an sich trug, gab an, daß die Schmerzen, die er als außergewöhnlich heftige schilderte, nur in der Ruhe bestehen. Stehen und längeres Sitzen machten ihm die größten Schwierigkeiten. Wenn er sich bewege, z. B. beim Tanzen, hören die Schmerzen vollständig auf. Auch hier fand sich am rechten Unterschenkel eine Abstumpfung der Sensibilität. Die genaue Umgrenzung der Sensibilitätsstörung kann ich leider nicht angeben, da ich den Kranken nur einmal flüchtig untersuchte. Eine Nachprüfung war mir bisher nicht möglich, da der Kranke sich seit Monaten außerhalb Münchens befindet. Dieser Kranke zeigte eine deutliche Rigidität der Nackenmuskulatur, des rechten Armes, in geringem Grade auch des rechten Beines, ferner einen zweifelhaften Oppenheim rechts. Sonst neurologisch nichts Bemerkenswertes.

Der dritte Fall betrifft eine 25jährige Näherin, die ebenfalls im März 1920 eine schwere Encephalitis durchmachte. Äußerlich bot auch sie das Bild einer Paralysis agitans. Sonst außer lebhaften Reflexen nichts Besonderes. Sie klagte über Schmerzen entlang und zu beiden Seiten der Wirbelsäule. Entsprechend ihrer Lokalisation bestand eine ausgesprochene Hyperästhesie für sämtliche Qualitäten (s. Abb. 3.) In diesem Falle war die Abhängigkeit von der Bewegung keine so eindeutige wie in den beiden ersten Fällen. Die Schmerzen wurden zwar wesentlich leichter durch die Bewegung und waren am stärksten im Stehen. Im Liegen dagegen hatte die Patientin überhaupt keine Schmerzen. Rumpfbeugung linderte die Schmerzen, Überstreckung der Wirbelsäule verstärkte sie. Im Gegensatz zu den beiden ersten Fällen bestanden die Schmerzen nicht von Beginn der Krankheit an, sie

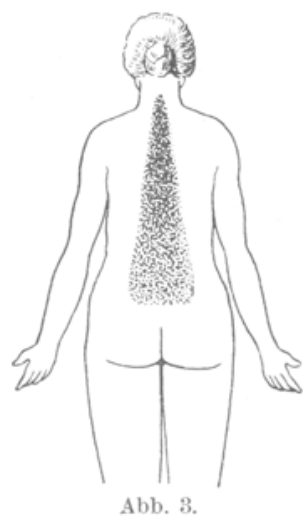

Hyperästhesie für sämtliche Qualitäten. stellten sich vielmehr erst während der $1 \mathrm{Jahr}$ später eingetretenen Schwangerschaft ein und haben an Stärke besonders seit der Entbindung zugenommen, die auch eine erhebliche Verschlech. terung ihres Allgemeinzustandes zur Folge hatte.

In dem letztgenannten Falle ist die zentrale Natur der Schmerzen zumal im Hinblick auf ihre Entstehungsgeschichte zum mindesten 
eine zweifelhafte. Jedenfalls handelt es sich offensichtlich nicht um cerebrale Störungen. In den beiden ersten Fällen ist dagegen ein Zweifel in dieser Richtung kaum möglich. Sie zeigen einen Schmerztypus, wie ich ihn bisher noch nirgends beschrieben gefunden habe: die konstante Abhängigkeit von der Bewegung im Sinne eines ausgesprochenen Ruheschmerzes, der mit Einsetzen jeder aktiven oder passiven Bewegung desjenigen Körperteiles, in den der Schmerz projiziert wird, prompt verschwindet, im ersteren Falle dazu noch eine ebenso konstante Abhängigkeit von der Atmung im Sinne eines Aussetzens der Schmerzen bei Atemstillstand. In allen 3 Fällen waren auch objektiv nachweisbare Sensibilitätsstörungen vorhanden. Und besonders hervorheben möchte ich, daß in keinem Falle Contracturen bestanden, in dem ersten Falle nicht einmal eine nennenswerte Rigidität. Ich erwähne es, weil die meisten Autoren (Cazauvielh, Sharkey u. a.) die schon früher bei cerebralen Affektionen anderer Ätiologie beobachteten Schmerzen und Parästhesien auf Spannungen und Zerrungen durch die Rigidität und Contracturen zurückführten, so daß $H$. Vogt, der sich darauf beruft, ihre zentrale Natur überhaupt für zweifelhaft hältt ${ }^{1}$ ). Eine Auffassung, die durch meine Fälle jedenfalls keine Stütze erfährt. Ganz abgesehen von dem Fehlen von Contracturen und von der Umgrenzung der objektiv nachweisbaren Sensibilitätsstörungen beweist meines Erachtens schon der oben beschriebene Schmerztypus die zentrale Natur der Schmerzen. Es ist zuzugeben, daß auch periphere Schmerzen durch aktive oder passive Bewegungen gelindert oder vorübergehend sogar vollständig beseitigt werden können - in der Regel dürften Bewegungen bei peripheren Erkrankungen eine Zunahme der Schmerzen zur Folge haben. Diese konstante Abhängigkeit von der Bewegung, wie ich sie oben geschildert habe, dürfte jedenfalls kaum jemals bei einer peripheren Erkrankung zur Beobachtung kommen. Und vollends ist die Beeinflussung der Schmerzen durch die Atmung an einem von ihr völlig unbeteiligten Körperteil nur denkbar unter der Voraussetzung ihres zentralen, im besonderen cerebralen Ursprungs. Ich erwähne noch, daß auch von seiten der Knochen, Bänder, Gelenke, Muskeln und Nerven jeder Anhaltspunkt zur Annahme einer peripheren Erkrankung fehlte. Die zentrale Natur der Schmerzen in den beiden ersten Fällen kann somit als feststehend angesehen werden.

Eine Verwechslung mit hysterischen Schmerzen, die im weiteren Sinne des Wortes ja auch zentrale sind, kommt ernstlich nur dann in Frage, wenn das klinische Bild sonst nur wenig ausgeprägt ist. Liegt aber einmal ein ausgesprochenes Pallidumsyndrom vor, so kann man getrost die Hysterie ausschließen. Unvergeßlich bleibt mir ein Kranker, der ursprünglich ein ausgesprochener Hysteriker, nach seiner Erkrankung

1) Lewandowsky, Handb. d. Neurol. 3, 306/07. 
an Encephalitis dann das übliche Bild einer lebenden Statue darbot. Die originäre prämorbide Persönlichkeit war wie ausgelöscht. Auch das Scopolamin, das seine pallidären Symptome zwar nicht beseitigte, aber doch merklich milderte, vermochte daran nichts zu ändern. Die „Hysterie" kam nicht wieder zum Vorschein. Es ist das auch nicht verwunderlich, wenn man bedenkt, daß es wohl keine schärferen Gegensätze gibt als einen versteinerten Pallidumkranken mit seiner Erschwerung und Verlangsamung aller Bewegungen, vor allem mit seinen auf ein Minimum eingeschränkten Reaktiv- und Ausdrucksbewegungen und einen Hysteriker, bei dem man nur anzutippen braucht, um einen Kurzschluß herbeizuführen. Ich dürfte wohl kaum zu weit gehen, wenn ich behaupte, daß hysterische Reaktionen ohne ein einigermaßen intaktes Pallidum schlechterdings unmöglich sind. Die Frage der Persönlichkeitswandlung unter dem Einfluß der Encephalitis ist ja ein ungemein reizvolles Problem, zu dem in neuerer Zeit Mayer-Gross und Steiner ${ }^{1}$ ) aus der Heidelberger Klinik und M. Kirschbaum ${ }^{1}$ ) aus der Kölner Klinik interessante Beiträge geliefert haben, und das wert wäre, genauer studiert zu werden.

Was nun den Sitz der den Schmerzen zugrunde liegenden Störungen anbelangt, so wird man bei dem gegenwärtigen Stande unseres Wissens in vivo über Vermutungen kaum hinauskommen. Die Annahme einer rein pallidären Störung hat wenig Wahrscheinlichkeit für sich, da man sonst Schmerzen bei Erkrankungen, die in so elektiver Weise wie die Encephalitis epidemica das Pallidum befallen, weit häufiger beobachten müßte, als es der Fall ist. Es ist daher weit naheliegender, an eine Lokalisation außerhalb desselben zu denken. Bekanntlich hat man zentrale Schmerzen hauptsächlich bei Erkrankungen des Thalamus gefunden. Seine topographische Lage und der Umstand, daß bei der Encephalitis epidemica in der Regel auch im Thalamus pathologisch-anatomische Veränderungen gefunden werden, läßt auch in den vorliegenden Fällen mit dieser Möglichkeit rechnen. In zweiter Linie kommt eine Affektion der inneren Kapsel, vor allem ihres hintersten Abschnittes, des Carrefour sensitif, in Frage. In diese Richtung weist im ersten Falle der, wenn auch nicht konstante Babinski, im zweiten der zweifelhafte Oppenheim. Nun ist es mir aber nicht bekannt, da $\beta$ bei Thalamusschmerzen, wie bei den bisher beobachteten zentralen Schmerzen überhaupt, jemals eine derartige Abhängigkeit von der Bewegung bzw. von der Atmung beobachtet worden wäre. In der Literatur habe ich nichts Derartiges gefunden. Die völlige Analogie des Ruheschmerzes mit dem Ruhetremor der Paralysis agitans,

1) Zeitschr. f. (1. ges. Neurol. u. Psychiatr. 73, Heft 4/5. 1921. 
der arterio-sklerotischen Muskelstarre und anderer exquisit pallidärer Erkrankungen drängt einem unwillkürlich den Gedanken auf, daß dieser Schmerztypus doch nicht ganz ohne Beziehungen zum Pallidum sein dürfte. Vielleicht hat die Deutung die größte Wahrscheinlichkeit für sich, daß die Schmerzen an sich durch eine extrapallidäre Komplikation - in Betracht kommen im Hinblick auf die Topographie nur der Thalamus und die Capsula interna - bedingt seien und die Miterkrankung des Pallidums ihnen nur die besondere Eigentümlichkeit des Ruheschmerzes verleihe.

Schließt man sich meinen obigen Ausführungen an, so wird man auch zur Frage der Sensibilitätsstörungen bei der Paralysis agitans, die symptomatologisch und lokalisatorisch den pallidären Formen der Encephalitis am nächsten steht, eine andere Stellung einnehmen müssen, als es bisher üblich war. Schmerzen sind zwar bei der Paralysis agitans kein konstantes und pathognomonisches Symptom, aber auch keine Seltenheit. Gibt es doch Fälle, in denen sie sogar im Vordergrunde der Beschwerden stehen, und bekanntlich ist sogar eine „Forme douloureuse" von L' Hirondel u. a. beschrieben worden ${ }^{1}$ ). Es steht mir leider augenblicklich kein entsprechendes Material zur Verfügung, um die Frage beantworten zu können, ob auch die Schmerzen bei der Paralysis agitans den Typus des Ruheschmerzes bzw. eine Abhängigkeit von der Atmung zeigen. Auf Grund meiner oben wiedergegebenen Erfahrungen vermute ich, daß es sich auch bei der Paralysis agitans in der Regel nicht um periphere, sondern um zentrale Schmerzen handelt $^{2}$ ). Abgesehen von Schmerzen sind bei der Paralysis agitans auch andere Sensibilitätsstörungen, neben den nicht seltenen Parästhesien auch Herabsetzung des Drucksinnes (Naumann), Hypästhesien und Hypalgesien und völlige Anästhesien beschrieben worden. Die meisten Autoren (Parkinson, Charcot, Gowers, Wollenberg, Strümpell, Oppenheim) sind allerdings der Ansicht, daß objektiv nachweisbare Störungen der Sensibilität nicht zum Bilde der Paralysis agitans gehören ${ }^{3}$ ). Es mag das in dem Sinne richtig sein, daß sie bei ausschließlicher Lokalisation des Krankheitsprozesses im Pallidum nicht vorkommen. Sehr unwahrscheinlich aber ist meines Erachtens die Auffassung, wie sie von Klieneberger vertreten wird, der die in seinen Fällen beobachteten Sensibilitätsstörungen als eine hysterische Beimengung deutet. Es ergibt sich dies ohne weiteres aus dem oben Gesagten.

1) Siche Oppenheim, Lehrbuch.

2) Eine willkommene Bestatigung meiner Vermutung, brachte mir die Diskussionsbemerkung des Kollegen Lissmann. Er berichtete von seinem an Paralysis agitans leidenden Vater, daß er seine Schmerzen am rechten Arm ebenso prompt wie die ersterwähnte Pat. durch Atemstillstand oder Bewegungen zum Verschwinden zu bringen gelernt habe.

3) Forster, Paralysis agitans in Lewandowskys Handbuch 3, 948. 
Nach dieser kleinen Abweichung zum Schlusse noch ein paar Worte über die Prognose und Therapie. Die Prognose der encephalitischen Schmerzen, soweit sie zentraler Natur sind, deckt sich wohl mit der des Grundleidens, ist also nach alledem, was wir bisber gesehen haben, quoad sanationem eine zum mindesten sehr zweifelhafte. Das einzige Mittel, das ich zu therapeutischen Zwecken bisher versucht habe, ist das bereits erwähnte Scopolamin, das ja auch sonst bei den Folgezuständen der Encephalitis als Symptomaticum gute Dienste leistet. Bezeichnenderweise konnten nur im Falle III die Schmerzen durch Scopolamin vollständig behoben werden und traten auch dann nicht auf, wenn mit dem Mittel einige Tage ausgesetzt wurde. Nur die objektiv nachweisbare Sensibilitätsstörung blieb unverändert bestehen. Im Falle II trat eine geringe Linderung ein, Fall I blieb trotz Überschreitung der Maximaldosis in bezug auf die Schmerzen völlig unbeeinflußt. 